(1)

trobertivier Journal of Nonlinear Mathematical Physics

\title{
Nonlocal symmetries and conservation laws of the Sinh-Gordon equation
}

Xiao-yan Tang, Zu-feng Liang

To cite this article: Xiao-yan Tang, Zu-feng Liang (2017) Nonlocal symmetries and conservation laws of the Sinh-Gordon equation, Journal of Nonlinear Mathematical Physics 24:1, 93-106, DOI: https://doi.org/10.1080/14029251.2017.1282246

To link to this article: https://doi.org/10.1080/14029251.2017.1282246

Published online: 04 January 2021 


\title{
Nonlocal symmetries and conservation laws of the Sinh-Gordon equation
}

\author{
Xiao-yan Tang* \\ Shanghai Key Laboratory of Trustworthy Computing, East China Normal University \\ Shanghai, 200062, China \\ xytang@sist.ecnu.edu.cn \\ Zu-feng Liang \\ Department of Physics, Hangzhou Normal University \\ Hangzhou 310036, China \\ liangzufeng@163.com
}

Received 16 March 2016

Accepted 17 December 2016

\begin{abstract}
Nonlocal symmetries of the (1+1)-dimensional Sinh-Gordon $(\mathrm{ShG})$ equation are obtained by requiring it, together with its Bäcklund transformation (BT), to be form invariant under the infinitesimal transformation. Naturally, the spectrum parameter in the BT enters the nonlocal symmetries, and thus through the parameter expansion procedure, infinitely many nonlocal symmetries of the ShG equation can be generated accordingly. Making advantages of the consistent conditions introduced when solving the nonlocal symmetires, some new nonlocal conservation laws of the ShG equation related to the nonlocal symmetries are obtained straightforwardly. Finally, taking the nonlocal symmetries as symmetry constraint conditions imposing on the BT, some new finite and infinite dimensional nonlinear systems are constructed.
\end{abstract}

Keywords: Nonlocal symmetry; Nonlocal conservation law; Bäcklund transformation; Sinh-Gordon equation.

2000 Mathematics Subject Classification: 70S10, 35Q53, 76M60

\section{Introduction}

Symmetry and symmetry related problems such as the conservation laws, similarity reductions, etc., are important topics of nonlinear systems especially for those integrable systems [1,25]. Many methods have been established to explore symmetries of nonlinear differential equations. For instance, the classical and the nonclassical Lie group approaches [2,26], and the CK's direct method [7,15,21] are the most famous and effective methods for finding Lie point symmetries. A new method was later developed to directly find finite symmetry transformation groups for Lax integrable nonlinear physical systems by considering the integrable system and its Lax pair to be form invariant under a type of symmetry group transformation, and then point symmetry algebras can be readily obtained. It has been realized for many systems such as the Kadomtsev Petviashvili equation, the dispersive long wave equation and the extended self dual Yang Mills equations [22]. More recently, the Gardner method which is traditionally utilized to find conservation laws of integrable equations has been generalized to generate the infinite hierarchy of symmetries of integrable equations, and its relation with the Lenard recursion has been also discussed [28].

*Corresponding author. 
Nonlocal symmetries were first studied rigorously early in 1980 by Vinogradov and Krasil'shchik [31] in which a satisfactory geometric formulation was developed by taking into account the requirement that a symmetry should transform solutions into solutions. The theory of coverings over differential equations was later established to describe various nonlocal phenomena including the nonlocal symmetries and conservation laws [14]. These nonlocal symmetries are the generalized symmetries by including pseudopotentials of the partial differential equations which are defined through the first order differential equations [8]. Some aspects of nonlocal symmetries were reviewed in [29] in a form as concrete as possible and some preliminary remarks were also made on their relation with other types of symmetries. Systematic procedures have been presented for finding nonlocally related partial differential equations and their many local and nonlocal conservation laws and nonlocal symmetries [3-6].

Nonlocal symmetries are of interest in that they are associated with the existence of linearizing transformations, Bäcklund transformations and Darboux transformations, and that they can also be used to construct highly nontrivial families of solutions $[11,29]$. In order to construct symmetry invariant solutions with nonlocal symmetries, the idea of "localizing" nonlocal symmetries by introducing auxiliary functions has been used to generate local symmetries of the augmented system [14]. Therefore, how to find suitable auxiliary function to localize nonlocal symmetries becomes a key point in finding group invariant solutions. For instance, applying the infinitesimal transformation on the nonlinear system and its Lax pair simultaneously, some useful nonlocal symmetries involving the eigenfunction can be obtained. These nonlocal symmetries, also known as the eigenfunction symmetries $[16,17,27]$, have been recently studied to construct explicit solutions $[9,12]$. It is found that nonlocal symmetries related to the Darboux and Bäcklund transformations can be localized, and thus similarity solutions, including the novel soliton-cnoidal wave solutions, were obtained $[10,23,30]$. In addition, nonlocal conservation laws related to nonlocal symmetries can also be constructed [32].

In this paper, we first look for nonlocal symmetries and the related nonlocal conservation laws for the (1+1)-dimensional Sinh-Gordon ( $\mathrm{ShG}$ ) equation; and then search for new integrable systems via the nonlocal symmetries. The (1+1)-dimensional $\mathrm{ShG}$ equation reads

$$
u_{x t}=\sinh u,
$$

which has an auto-Bäcklund transformation (BT)

$$
\begin{aligned}
& u_{x}+v_{x}=-4 \lambda \sinh \left(\frac{u}{2}-\frac{v}{2}\right), \\
& u_{t}-v_{t}=-\frac{1}{\lambda} \sinh \left(\frac{u}{2}+\frac{v}{2}\right) .
\end{aligned}
$$

That is to say, if $u$ is a solution of the ShG equation (1.1), then $v$ determined by the BT (1.2)-(1.3) also satisfies the $\mathrm{ShG}$ equation in the form of

$$
v_{x t}=\sinh v .
$$

In the next section, nonlocal symmetries related to the BT (1.2)-(1.3) for the ShG equation (1.1) are solved from the linearized system of the ShG equation and its BT, which are obtained by requiring these equations form invariant under the infinitesimal transformation. Nonlocal conservation laws related to the nonlocal symmetries are then presented in section 3. Then taking the nonlocal symmetries as symmetry constraint conditions, some new finite and infinite dimensional nonlinear 
systems are constructed in section 4 . In section 5 , the general Lie point symmetry of the enlarged system is presented, from which two types of similarity solutions of the ShG equation are obtained. The last section is devoted to summary and discussions.

\section{Nonlocal symmetries related to BT}

To find nonlocal symmetries of the ShG equation (1.1) related to its BT (1.2)-(1.3), one has to solve the linearized equations of the ShG equation together with its BT, which are obtained by requiring these equations form invariant under the following infinitesimal transformation

$$
u \rightarrow u+\varepsilon \sigma^{u}, \quad v \rightarrow v+\varepsilon \sigma^{v}, \quad \lambda \rightarrow \lambda+\varepsilon \delta,
$$

with $\varepsilon$ being an infinitesimal parameter. Direct calculations arrive at the linearized system for symmetries $\sigma^{u}$ and $\sigma^{v}$

$$
\begin{aligned}
& \sigma_{x t}^{u}-m^{2} \cos (u) \sigma^{u}=0, \\
& \sigma_{x t}^{v}-m^{2} \cos (v) \sigma^{v}=0, \\
& \sigma_{x}^{u}-\sigma_{x}^{v}-2 \delta m \sin \left(\frac{u}{2}+\frac{v}{2}\right)-\lambda m \cos \left(\frac{u}{2}+\frac{v}{2}\right)\left(\sigma^{u}+\sigma^{v}\right)=0, \\
& \sigma_{t}^{u}+\sigma_{t}^{v}+2 \frac{m \delta}{\lambda^{2}} \sin \left(\frac{u}{2}-\frac{v}{2}\right)-\frac{m}{\lambda} \cos \left(\frac{u}{2}-\frac{v}{2}\right)\left(\sigma^{u}-\sigma^{v}\right)=0 .
\end{aligned}
$$

Solving the above linear differential equations (2.2)-(2.5), we find three special solutions.

(1). If $\delta=0$ and $\sigma^{v}=0$, then we have the nonlocal symmetry $\sigma^{u}$ given by

$$
\sigma_{1}^{u}=e^{-2 \lambda p}
$$

where $p$ is determined by

$$
p_{x}=\cosh \left(\frac{u}{2}-\frac{v}{2}\right), \quad p_{t}=\frac{1}{4 \lambda^{2}} \cosh \left(\frac{u}{2}+\frac{v}{2}\right),
$$

and $p_{x t}=p_{t x}$ is satisfied identically via the BT (1.2)-(1.3).

(2). If $\delta=1 / 2 m$ and $\sigma^{v}=0$, then the second special nonlocal symmetry reads

$$
\sigma_{2}^{u}=q e^{-2 \lambda p}
$$

where $p$ satisfies Eq. (2.7), and $q$ is given by

$$
q_{x}=e^{2 \lambda p} \sinh \left(\frac{u}{2}-\frac{v}{2}\right), \quad q_{t}=-\frac{1}{4 \lambda^{2}} \sinh \left(\frac{u}{2}+\frac{v}{2}\right) e^{2 \lambda p},
$$

with $q_{x t}=q_{t x}$ satisfied identically by utilizing the BT (1.2)-(1.3).

(3). When $\sigma^{v}=v_{x}$ and $\delta=0$, the third possible nonlocal symmetry can be obtained as

$$
\sigma_{3}^{u}=v_{x}+2 m \lambda f e^{\lambda m p},
$$

where $p$ satisfies Eq. (2.7), $f$ is determined by

$$
f_{x}=v_{x} \cos \left(\frac{u}{2}+\frac{v}{2}\right) e^{-\lambda m p}, \quad f_{t}=-\lambda m v_{x t} e^{-\lambda m p},
$$

and the consistent condition $f_{x t}=f_{t x}$ is also satisfied identically by the use of the ShG equation (1.4) and the BT (1.2)-(1.3). 
It is evident that symmetries $\sigma^{u}$ of the ShG equation (1.1) obtained above are really nonlocal as they depend on the function $v$, which is related to the function $u$ through BT (1.2)-(1.3). In addition, these nonlocal symmetries are parameter dependent, which makes it possible to obtain infinitely many nonlocal symmetries by expanding the symmetries around the neighborhood of the spectrum parameter $\lambda$. The procedure is straightforward. A series of infinite number of nonlocal symmetries from the nonlocal symmetry (2.8) is presented in the following, and another two series of infinitely many nonlocal symmetries can be written down in a similar way.

As mentioned above, to construct infinitely many nonlocal symmetries from the nonlocal symmetry (2.8), one should expand the functions $p$ and $q$ as

$$
p=\sum_{i=0}^{\infty} p_{i} \delta^{i}, \quad q=\sum_{i=0}^{\infty} q_{i} \delta^{i}
$$

where the expansion coefficients $p_{i}, q_{i}$ are functions of $x$ and $t$, and $\delta$ is an arbitrary expansion constant. Substituting the expansions (2.12) into the nonlocal symmetry (2.8) with $\lambda$ replaced by $\lambda+\delta$ gives

$$
\sigma_{2}^{u}=\left(\sum_{i=0}^{\infty} q_{i} \delta^{i}\right) \exp \left(-2(\lambda+\delta) \sum_{i=0}^{\infty} p_{i} \delta^{i}\right) \equiv \sum_{i=0}^{\infty} \sigma_{2 i}^{u} \delta^{i},
$$

where $\sigma_{2 i}^{u}$ is determined by

$$
\sigma_{2 i}^{u}=\left.\frac{d^{n} \sigma_{2}^{u}}{d \delta^{n}}\right|_{\delta=0}
$$

It is easy to verify that all the expansion coefficients $\sigma_{2 i}^{u}$ are nonlocal symmetries, and thus infinitely many nonlocal symmetries $\sigma_{2 i}^{u}$ are produced from the nonlocal symmetry (2.8). However, it is not easy to write down the general explicit expression for the series of infinity number of nonlocal symmetries $\sigma_{2 i}^{u}$. Therefore, one has to write down the explicit forms one by one from the general expansion formula. To do so, the function $v$ needs the similar parameter expansion as it also appears both in Eqs. (2.7), (2.9) and the BT (1.2)-(1.3). After some simple and straightforward calculations, the explicit descriptions for the nonlocal symmetries $\sigma_{2 i}^{u}$ for $i=1,2,3$ can be found as

$$
\begin{aligned}
\sigma_{21}^{u}= & \left(q_{1}-2 q_{0}\left(p_{0}+\lambda p_{1}\right)\right) e^{-2 \lambda p_{0}}, \\
\sigma_{22}^{u}= & \left(q_{2}-2 q_{1}\left(p_{0}+\lambda p_{1}\right)+2 q_{0}\left(\left(p_{0}+\lambda p_{1}\right)^{2}-\left(p_{1}+\lambda p_{2}\right)\right)\right) e^{-2 \lambda p_{0}}, \\
\sigma_{23}^{u}= & {\left[q_{3}-2 q_{2}\left(p_{0}+\lambda p_{1}\right)+2 q_{1}\left(\left(p_{0}+\lambda p_{1}\right)^{2}-\left(p_{1}+\lambda p_{2}\right)\right)-2 q_{0}\left(\frac{2}{3}\left(p_{0}+\lambda p_{1}\right)^{3}\right.\right.} \\
& \left.\left.-2\left(p_{0}+\lambda p_{1}\right)\left(p_{1}+\lambda p_{2}\right)+\left(p_{2}+\lambda p_{3}\right)\right)\right] e^{-2 \lambda p_{0}},
\end{aligned}
$$

where $p_{i}, q_{i}(i=0,1,2,3,4)$ are determined by

$$
\begin{gathered}
p_{0 x}=\cosh \left(\frac{u}{2}-\frac{v_{0}}{2}\right), \\
p_{0 t}=\frac{1}{4 \lambda^{2}} \cosh \left(\frac{u}{2}+\frac{v_{0}}{2}\right), \\
p_{1 x}=-\frac{1}{2} v_{1} \sinh \left(\frac{u}{2}-\frac{v_{0}}{2}\right), \\
p_{1 t}=-\frac{1}{2 \lambda^{3}} \cosh \left(\frac{u}{2}+\frac{v_{0}}{2}\right)+\frac{1}{8 \lambda^{2}} v_{1} \sinh \left(\frac{u}{2}+\frac{v_{0}}{2}\right), \\
\text { Co-published by Atlantis Press and Taylor \& Francis } \\
\text { Copyright: the authors }
\end{gathered}
$$




$$
\begin{aligned}
& p_{2 x}=\frac{1}{8} v_{1}^{2} \cosh \left(\frac{u}{2}-\frac{v_{0}}{2}\right)-\frac{1}{2} v_{2} \sinh \left(\frac{u}{2}-\frac{v_{0}}{2}\right), \\
& p_{2 t}=\left(\frac{3}{4 \lambda^{4}}+\frac{v_{1}^{2}}{32 \lambda^{2}}\right) \cosh \left(\frac{u}{2}+\frac{v_{0}}{2}\right)-\left(\frac{v_{1}}{4 \lambda^{3}}-\frac{v_{2}}{8 \lambda^{2}}\right) \sinh \left(\frac{u}{2}+\frac{v_{0}}{2}\right), \\
& p_{3 x}=\frac{1}{4} v_{1} v_{2} \cosh \left(\frac{u}{2}-\frac{v_{0}}{2}\right)-\frac{1}{48}\left(v_{1}^{3}+24 v_{3}\right) \sinh \left(\frac{u}{2}-\frac{v_{0}}{2}\right), \\
& p_{3 t}=\left(\frac{v_{1} v_{2}}{16 \lambda^{2}}-\frac{v_{1}^{2}}{16 \lambda^{3}}-\frac{1}{\lambda^{5}}\right) \cosh \left(\frac{u}{2}+\frac{v_{0}}{2}\right) \\
& +\left(\frac{24 v_{3}+v_{1}^{3}}{192 \lambda^{2}}-\frac{v_{2}}{4 \lambda^{3}}+\frac{3 v_{1}}{8 \lambda^{4}}\right) \sinh \left(\frac{u}{2}+\frac{v_{0}}{2}\right) \\
& q_{0 x}=\sinh \left(\frac{u}{2}-\frac{v_{0}}{2}\right) e^{2 \lambda p_{0}} \\
& q_{0 t}=-\frac{1}{4 \lambda^{2}} \sinh \left(\frac{u}{2}+\frac{v_{0}}{2}\right) e^{2 \lambda p_{0}} \\
& q_{1 x}=-\frac{v_{1}}{2} \cosh \left(\frac{u}{2}-\frac{v_{0}}{2}\right) e^{2 \lambda p_{0}}+2\left(p_{0}+\lambda p_{1}\right) \sinh \left(\frac{u}{2}-\frac{v_{0}}{2}\right) e^{2 \lambda p_{0}}, \\
& q_{1 t}=-\frac{v_{1}}{8 \lambda^{2}} \cosh \left(\frac{u}{2}+\frac{v_{0}}{2}\right) e^{2 \lambda p_{0}}-\frac{\lambda^{2} p_{1}+\lambda p_{0}-1}{2 \lambda^{3}} \sinh \left(\frac{u}{2}+\frac{v_{0}}{2}\right) e^{2 \lambda p_{0}}, \\
& q_{2 x}=-\frac{1}{2}\left(2 v_{1}\left(p_{0}+\lambda p_{1}\right)+v_{2}\right) \cosh \left(\frac{u}{2}-\frac{v_{0}}{2}\right) e^{2 \lambda p_{0}} \\
& +\frac{1}{8}\left(v_{1}^{2}+16\left(p_{0}+\lambda p_{1}\right)^{2}+16\left(p_{1}+\lambda p_{2}\right)\right) \sinh \left(\frac{u}{2}-\frac{v_{0}}{2}\right) e^{2 \lambda p_{0}}, \\
& q_{2 t}=\left(-\frac{v_{1} p_{1}}{4 \lambda}-\frac{2 v_{1} p_{0}+v_{2}}{8 \lambda^{2}}+\frac{v_{1}}{4 \lambda^{3}}\right) \cosh \left(\frac{u}{2}+\frac{v_{0}}{2}\right) e^{2 \lambda p_{0}}-\left(\frac{p_{1}^{2}}{2}+\frac{p_{2}+2 p_{0} p_{1}}{2 \lambda}\right. \\
& \left.+\frac{v_{1}^{2}-16 p_{1}-16 p_{0}^{2}}{32 \lambda^{2}}-\frac{p_{0}}{\lambda^{3}}+\frac{3}{4 \lambda^{4}}\right) \sinh \left(\frac{u}{2}+\frac{v_{0}}{2}\right) e^{2 \lambda p_{0}} \\
& q_{3 x}=-\left(v_{1}\left(p_{0}+\lambda p_{1}\right)^{2}+v_{2}\left(p_{0}+\lambda p_{1}\right)+v_{1}\left(p_{1}+\lambda p_{2}\right)+\frac{v_{1}^{3}}{48}+\frac{v_{3}}{2}\right) \cosh \left(\frac{u}{2}-\frac{v_{0}}{2}\right) e^{2 \lambda p_{0}} \\
& +\left(\frac{4}{3}\left(p_{0}+\lambda p_{1}\right)^{3}+\frac{1}{4}\left(v_{1}^{2}+16 \lambda p_{2}+16 p_{1}\right)\left(p_{0}+\lambda p_{1}\right)+2\left(p_{2}+\lambda p_{3}\right)\right. \\
& \left.+\frac{1}{4} v_{1} v_{2}\right) \sinh \left(\frac{u}{2}-\frac{v_{0}}{2}\right) e^{2 \lambda p_{0}} \\
& q_{3 t}=\left(-\frac{1}{4} v_{1} p_{1}^{2}-\frac{2 v_{1} p_{0} p_{1}+v_{2} p_{1}+v_{1} p_{2}}{4 \lambda}-\frac{v_{1} p_{0}^{2}+v_{2} p_{0}-v_{1} p_{1}}{4 \lambda^{2}}-\frac{v_{1}^{3}}{192 \lambda^{2}}-\frac{v_{3}}{8 \lambda^{2}}\right. \\
& \left.+\frac{2 v_{1} p_{0}+v_{2}}{4 \lambda^{3}}-\frac{3 v_{1}}{8 \lambda^{4}}\right) \cosh \left(\frac{u}{2}+\frac{v_{0}}{2}\right) e^{2 \lambda p_{0}}+\left(-\frac{1}{3} p_{1}^{3} \lambda-p_{1}\left(p_{0} p_{1}+p_{2}\right)\right. \\
& -\frac{v_{1}^{2} p_{1}+16 p_{0}^{2} p_{1}+16 p_{2} p_{0}+8 p_{3}}{16 \lambda}-\frac{v_{1}^{2} p_{0}+v_{1} v_{2}}{16 \lambda^{2}}-\frac{2 p_{0}^{3}-6 p_{0} p_{1}-3 p_{2}}{6 \lambda^{2}} \\
& \left.+\frac{v_{1}^{2}+16 p_{0}^{2}-8 p_{1}}{16 \lambda^{3}}-\frac{3 p_{0}}{2 \lambda^{4}}+\frac{1}{\lambda^{5}}\right) \sinh \left(\frac{u}{2}+\frac{v_{0}}{2}\right) e^{2 \lambda p_{0}},
\end{aligned}
$$


respectively, yielded by inserting the expansions (2.12) and

$$
v=\sum_{i=0}^{\infty} v_{i} \delta^{i}
$$

into Eqs. (2.7) and (2.9) with $\lambda$ replaced by $\lambda+\delta$, and then equating zero all the coefficients of different powers of $\delta$. The $v_{i}(i=0,1,2,3)$ in Eqs. (2.18)-(2.34) are similarly obtained to satisfy

$$
\begin{gathered}
v_{0 x}=-u_{x}-4 \lambda \sinh \left(\frac{u}{2}-\frac{v_{0}}{2}\right), \\
v_{0 t}=u_{t}+\frac{1}{\lambda} \sinh \left(\frac{u}{2}+\frac{v_{0}}{2}\right) \\
v_{1 x}=2 \lambda v_{1} \cosh \left(\frac{u}{2}-\frac{v_{0}}{2}\right)-4 \sinh \left(\frac{u}{2}-\frac{v_{0}}{2}\right), \\
v_{1 t}=\frac{1}{2 \lambda} v_{1} \cosh \left(\frac{u}{2}+\frac{v_{0}}{2}\right)-\frac{1}{\lambda^{2}} \sinh \left(\frac{u}{2}+\frac{v_{0}}{2}\right) . \\
v_{2 x}=2\left(v_{1}+\lambda v_{2}\right) \cosh \left(\frac{u}{2}-\frac{v_{0}}{2}\right)-\frac{1}{2} \lambda v_{1}^{2} \sinh \left(\frac{u}{2}-\frac{v_{0}}{2}\right), \\
v_{2 t}=\left(-\frac{v_{1}}{2 \lambda^{2}}+\frac{v_{2}}{2 \lambda}\right) \cosh \left(\frac{u}{2}+\frac{v_{0}}{2}\right)+\left(\frac{1}{\lambda^{3}}+\frac{v_{1}^{2}}{8 \lambda}\right) \sinh \left(\frac{u}{2}+\frac{v_{0}}{2}\right), \\
v_{3 x}=\left(\frac{1}{12} \lambda\left(v_{1}^{3}+24 v_{3}\right)+2 v_{2}\right) \cosh \left(\frac{u}{2}-\frac{v_{0}}{2}\right)-\frac{1}{2} v_{1}\left(v_{1}+2 \lambda v_{2}\right) \sinh \left(\frac{u}{2}-\frac{v_{0}}{2}\right), \\
\left(\frac{v_{1}^{3}+24 v_{3}}{48 \lambda}-\frac{v_{2}}{2 \lambda^{2}}+\frac{v_{1}}{2 \lambda^{3}}\right) \cosh \left(\frac{u}{2}+\frac{v_{0}}{2}\right) \\
+\left(\frac{v_{1} v_{2}}{4 \lambda}-\frac{v_{1}^{2}}{8 \lambda^{2}}-\frac{1}{4 \lambda^{4}}\right) \sinh \left(\frac{u}{2}+\frac{v_{0}}{2}\right)
\end{gathered}
$$

It is remarked that the consistent conditions $v_{i x t}=v_{i t x}(i=0,1,2,3)$ are all satisfied identically with the ShG equation (1.1), $p_{i x t}=p_{i t x}$ and $q_{i x t}=q_{i t x},(i=0,1,2,3)$ are all satisfied identically with $v_{i}(i=0,1,2,3)$ determined by Eqs. (2.36)-(2.43). It is also remarked that the symmetries determined by Eqs. (2.15)-(2.17) are all nonlocal symmetries of the ShG equation (1.1) which can by verified by their substitution with Eqs. (2.18)-(2.34) and (2.36)-(2.43) into the symmetry equation (2.2).

\section{Nonlocal conservation laws related to the nonlocal symmetries}

It is known that the conservation law of the ShG equation (1.1) must satisfy the identity

$$
\frac{\partial}{\partial t} \rho(x, t)+\frac{\partial}{\partial x} J(x, t)=0,
$$

for any solution $u$ of the ShG equation (1.1), where the functions $\rho$ and $J$ are called the conserved density and the conserved flux, correspondingly. This divergence expression (3.1) can be easily obtained from some auxiliary functions introduced when solving the symmetry equations (2.4) and (2.5). Several new special nonlocal conservation laws are presented in this section. 
Integrating Eqs. (2.4) and (2.5) with respect to $x$ and $t$, respectively, leads to

$$
\sigma^{u}=-4 e^{-2 \lambda p} q \delta+4 \lambda e^{-2 \lambda p} \int p_{x} \sigma^{v} e^{2 \lambda p} \mathrm{~d} x-\sigma^{v}+e^{-2 \lambda p} F_{1}(t),
$$

and

$$
\sigma^{u}=\frac{s}{\lambda^{2}} e^{-\frac{r}{2 \lambda}} \delta-\frac{1}{\lambda} e^{-\frac{r}{2 \lambda}} \int r_{t} \sigma^{v} e^{\frac{r}{2 \lambda}} \mathrm{d} t+\sigma^{\nu}+e^{-\frac{r}{2 \lambda}} F_{2}(x),
$$

respectively, where $p, q, r, s$ satisfy

$$
\begin{aligned}
p_{x} & =\cosh \left(\frac{u}{2}-\frac{v}{2}\right), \\
q_{x} & =\sinh \left(\frac{u}{2}-\frac{v}{2}\right) e^{2 \lambda p}, \\
r_{t} & =\cosh \left(\frac{u}{2}+\frac{v}{2}\right), \\
s_{t} & =\sinh \left(\frac{u}{2}+\frac{v}{2}\right) e^{-\frac{r}{2 \lambda}},
\end{aligned}
$$

$F_{1}(t)$ and $F_{2}(x)$ are arbitrary integration functions of the indicated variables. To Equal the above two integration results (3.2) and (3.3) with the help of the arbitrariness of the constant $\delta$ and integration functions $F_{1}, F_{2}$, three conditions

$$
\begin{gathered}
r=4 \lambda^{2} p, \\
s=-4 \lambda^{2} q,
\end{gathered}
$$

and

$$
\int p_{x} \sigma^{v} e^{2 \lambda p} \mathrm{~d} x+\int p_{t} \sigma^{v} e^{2 \lambda p} \mathrm{~d} t-\frac{1}{2 \lambda} \sigma^{v} e^{2 \lambda p}=0,
$$

should be satisfied, which straightforwardly lead to three nontrivial nonlocal conservation laws with the help of Eqs. (3.4)-(3.7). The corresponding conserved density and flux read

$$
\begin{gathered}
\rho_{1}=4 \lambda^{2} \cosh \left(\frac{u}{2}-\frac{v}{2}\right), \quad J_{1}=-\cosh \left(\frac{u}{2}+\frac{v}{2}\right), \\
\rho_{2}=\frac{4 \lambda^{2}}{\sigma_{1}^{u}} \sinh \left(\frac{u}{2}-\frac{v}{2}\right), \quad J_{2}=\frac{1}{\sigma_{1}^{u}} \sinh \left(\frac{u}{2}+\frac{v}{2}\right),
\end{gathered}
$$

and

$$
\rho_{3}=\frac{\lambda \sigma_{x}^{v}}{\sigma_{1}^{u}}, \quad J_{3}=-\frac{\sigma^{v}}{2 \sigma_{1}^{u}} \cosh \left(\frac{u}{2}+\frac{v}{2}\right),
$$

respectively, where $\sigma_{1}^{u}$ is given by Eq. (2.6), and $\sigma^{v}$ is the symmetry of the function $v$ determined by Eqs. (2.2)-(2.5).

It is noted that these nonlocal conservation laws are connected with the nonlocal symmetries and also parameter dependent, hence, they can generate infinitely many nonlocal conservation laws by means of the parameter expansion procedure. For instance, from the nonlocal conservation law (3.11), we can construct infinitely many nonlocal conservation laws $\partial_{t} \rho_{1 i}(x, t)+\partial_{x} J_{1 i}(x, t)=0, i=$ 
$0,1,2, \ldots$, by inserting the expansion (2.35) into the conserved density and flux with $\lambda$ replaced by $\lambda+\delta$ and then equalling zero the coefficients of the same orders of $\delta$. The first five nonlocal conserved density read

$$
\begin{gathered}
\rho_{10}=4 \lambda^{2} \cosh \left(\frac{u}{2}-\frac{v_{0}}{2}\right) \\
\rho_{11}=8 \lambda \cosh \left(\frac{u}{2}-\frac{v_{0}}{2}\right)-2 \lambda^{2} v_{1} \sinh \left(\frac{u}{2}-\frac{v_{0}}{2}\right) \\
\rho_{12}=\left(4+\frac{1}{2} \lambda^{2} v_{1}^{2}\right) \cosh \left(\frac{u}{2}-\frac{v_{0}}{2}\right)-2 \lambda\left(2 v_{1}+\lambda v_{2}\right) \sinh \left(\frac{u}{2}-\frac{v_{0}}{2}\right), \\
\rho_{13}=\lambda v_{1}\left(v_{1}+\lambda v_{2}\right) \cosh \left(\frac{u}{2}-\frac{v_{0}}{2}\right)-\left[\lambda^{2}\left(\frac{1}{12} v_{1}^{3}+2 v_{3}\right)+4 \lambda v_{2}+2 v_{1}\right] \sinh \left(\frac{u}{2}-\frac{v_{0}}{2}\right) \\
\rho_{14}=\left[\lambda^{2}\left(\frac{1}{96} v_{1}^{4}+\frac{1}{2} v_{2}^{2}+v_{1} v_{3}\right)+2 \lambda v_{1} v_{2}+\frac{1}{2} v_{1}^{2}\right] \cosh \left(\frac{u}{2}-\frac{v_{0}}{2}\right) \\
-\left[\lambda^{2}\left(\frac{1}{4} v_{1}^{2} v_{2}+2 v_{4}\right)+\lambda\left(\frac{1}{6} v_{1}^{3}+4 v_{3}\right)+2 v_{2}\right] \sinh \left(\frac{u}{2}-\frac{v_{0}}{2}\right),
\end{gathered}
$$

and the corresponding nonlocal conserved flux read

$$
\begin{gathered}
J_{10}=\cosh \left(\frac{u}{2}+\frac{v_{0}}{2}\right), \\
J_{11}=\frac{1}{2} v_{1} \sinh \left(\frac{u}{2}+\frac{v_{0}}{2}\right), \\
J_{12}=\frac{1}{8} v_{1}^{2} \cosh \left(\frac{u}{2}+\frac{v_{0}}{2}\right)+\frac{1}{2} v_{2} \sinh \left(\frac{u}{2}+\frac{v_{0}}{2}\right), \\
J_{13}=\frac{1}{4} v_{1} v_{2} \cosh \left(\frac{u}{2}+\frac{v_{0}}{2}\right)+\left(\frac{1}{48} v_{1}^{3}+\frac{1}{2} v_{3}\right) \sinh \left(\frac{u}{2}+\frac{v_{0}}{2}\right), \\
J_{14}=\left(\frac{1}{384} v_{1}^{4}+\frac{1}{8} v_{2}^{2}+\frac{1}{4} v_{1} v_{3}\right) \cosh \left(\frac{u}{2}+\frac{v_{0}}{2}\right)+\left(\frac{1}{16} v_{1}^{2} v_{2}+\frac{1}{2} v_{4}\right) \sinh \left(\frac{u}{2}+\frac{v_{0}}{2}\right),
\end{gathered}
$$

respectively, where $v_{i}(i=0,1,2,3,4)$ are determined by Eqs. (2.36)-(2.43).

It is remarked that the similar parameter expansion procedure can also be applied to the compatibility condition of the BT (1.2)-(1.3) to produce infinitely many nonlocal conservation laws of the ShG equation (1.1). In addition, one can also obtain infinitely many local conservation laws when expanding the functions around the neighbourhood of the parameter $\lambda=0$. In this case, some trivial local conservation laws need to be further excluded. 


\section{New integrable systems from nonlocal symmetry}

The symmetry constraint method is powerful to establish new integrable systems from known soliton equations, for instance, by imposing symmetry constraint conditions on their Lax pairs $[13,23,24]$. Here, the nonlocal symmetry constraints are applied to the BT to give some new integrable systems in lower and higher dimensions [23].

Take each pair $\left(u, u_{i}\right),(i=1,2, \ldots, N)$ satisfy the BT

$$
\begin{aligned}
& u_{x}+u_{i x}=-4 \lambda_{i} \sinh \left(\frac{u}{2}-\frac{u_{i}}{2}\right), \\
& u_{t}-u_{i t}=-\frac{1}{\lambda_{i}} \sinh \left(\frac{u}{2}+\frac{u_{i}}{2}\right),
\end{aligned}
$$

and the nonlocal symmetry of $u$ given by $\sigma^{i}=\exp \left(-2 \lambda_{i} \int \cosh \left(\frac{u}{2}-\frac{u_{i}}{2}\right) \mathrm{d} x\right),(i=1,2, \ldots, N)$.

\subsection{Finite dimensional nonlinear integrable systems}

A finite dimensional $(N+1)$-component integro-differential system

$$
\begin{aligned}
& u_{x}=\sum_{i=1}^{N} a_{i} \exp \left(-2 \lambda \int \cosh \left(\frac{u}{2}-\frac{u_{i}}{2}\right) \mathrm{d} x\right), \\
& u_{x}+u_{i x}=-4 \lambda_{i} \sinh \left(\frac{u}{2}-\frac{u_{i}}{2}\right), \quad i=1,2, \ldots, N,
\end{aligned}
$$

with arbitrary constants $a_{i}$ and $\lambda_{i}$ can be obtained by imposing the generalized symmetry constraint

$$
u_{x}=\sum_{i=1}^{N} a_{i} \exp \left(-2 \lambda_{i} \int \cosh \left(\frac{u}{2}-\frac{u_{i}}{2}\right) \mathrm{d} x\right)
$$

on the $x$-part of the BT (1.2). Taking

$$
u_{i}=u-2 \operatorname{arccosh}\left(-\frac{1}{2 \lambda_{i}}\left(\ln w_{i x}\right)_{x}\right)
$$

the symmetry constraint condition (4.5) can be simplified as

$$
u=\sum_{m=1}^{N} a_{m} w_{m}
$$

and thus transforms Eqs. (4.3)-(4.4) into a $N$-component differential system

$$
\left(s_{i x}^{2}-4 \lambda_{i}^{2} s_{i}^{2}\right)\left(\sum_{m=1}^{N} a_{m} s_{m}\right)^{2}-\left(s_{i x x}-4 \lambda_{i}^{2} s_{i}\right)^{2}=0, \quad i=1,2, \ldots, N .
$$

with $s_{i}=w_{i x}$.

It is noted that in the same way, the symmetry constraint (4.5) can also be applied to the $t$-part of the BT (1.3) to form another new integrable system, which seems to be much more complicated. 
However, a very similar $N$-component system

$$
4 \lambda_{i}^{2}\left(4 s_{i t}^{2} \lambda_{i}^{2}-s_{i}^{2}\right)\left(\sum_{m=1}^{N} a_{m} s_{m}\right)^{2}+\left(4 s_{i t t} \lambda_{i}^{2}-s_{i}\right)^{2}=0, \quad i=1,2, \ldots, N,
$$

can be obtained by adding the following symmetry constraint

$$
u_{t}=\sum_{i=1}^{N} a_{i} \exp \left(-\frac{1}{2 \lambda_{i}} \int \cosh \left(\frac{u}{2}+\frac{u_{i}}{2}\right) \mathrm{d} t\right)
$$

on the $t$-part of the BT (1.3)

$$
u_{t}-u_{i t}=-\frac{1}{\lambda_{i}} \sinh \left(\frac{u}{2}+\frac{u_{i}}{2}\right), \quad i=1,2, \ldots, N
$$

with the transformation $u_{i}=-u+2 \operatorname{arccosh}\left(-2 \lambda_{i}\left(\ln s_{i}\right)_{t}\right)$.

\subsection{Infinite dimensional nonlinear integrable systems}

Besides finite dimensional nonlinear systems, one may also construct some higher dimensional nonlinear models through the introduction of some internal parameters. In a similar way, some internal parameter dependent symmetry constraints can be applied on the BT to produce infinite dimensional nonlinear systems [18-20].

Now we can take

$$
u_{y}=\sum_{i=1}^{N} a_{i} \exp \left(-2 \lambda_{i} \int \cosh \left(\frac{u}{2}-\frac{u_{i}}{2}\right) \mathrm{d} x\right)
$$

as a new symmetry constraint condition, because the ShG equation (1.1) is invariant under the inner parameter $y$ translation. By using it on the $x$-part of the BT (1.2) leads to a (1+1)-dimensional $(N+1)$-component integro-differential system

$$
\begin{aligned}
& u_{y}=\sum_{i=1}^{N} a_{i} \exp \left(-2 \lambda_{i} \int \cosh \left(\frac{u}{2}-\frac{u_{i}}{2}\right) \mathrm{d} x\right), \\
& u_{x}+u_{i x}=-4 \lambda_{i} \sinh \left(\frac{u}{2}-\frac{u_{i}}{2}\right), \quad i=1,2, \ldots, N,
\end{aligned}
$$

with arbitrary constants $a_{i}$ and $\lambda_{i}$.

The above system of Eqs. (4.13)-(4.14) can be converted into a (1+1)-dimensional $N$-component differential system

$$
4\left(f_{i x y}^{2}-4 f_{i y}^{2} \lambda_{i}^{2}\right)\left(\sum_{m=1}^{N} a_{m} f_{m x}\right)^{2}+\left(f_{i x x y}-8 f_{i y} \lambda_{i}^{2}\right)^{2}=0,
$$

by means of the transformation

$$
u=u_{i}+2 \operatorname{arccosh}\left(-\frac{1}{2 \lambda_{i}}\left(\ln f_{y}\right)_{x}\right)=\sum_{m=1}^{N} a_{m} f_{m}
$$


If the symmetry condition (4.12) is similarly imposed on the $t$-part of the BT (1.3) as

$$
\begin{aligned}
& u_{y}=\sum_{i=1}^{N} a_{i} \exp \left(-2 \lambda_{i} \int \cosh \left(\frac{u}{2}-\frac{u_{i}}{2}\right) \mathrm{d} x\right), \\
& u_{t}-u_{i t}=-\frac{1}{\lambda_{i}} \sinh \left(\frac{u}{2}+\frac{u_{i}}{2}\right), \quad i=1,2, \ldots, N,
\end{aligned}
$$

then a complicated (2+1)-dimensional $N$-component differential system can be obtained as

$$
f_{i y}^{2}\left(f_{i x y}^{2}-4 f_{i y}^{2} \lambda_{i}^{2}\right) \sinh ^{2}\left(\sum_{m=1}^{N} a_{m} f_{m}-\operatorname{arccosh}\left(-\frac{f_{i x y}}{2 \lambda_{i} f_{i y}}\right)\right)-4 \lambda_{i}^{2}\left(f_{i y} f_{i x y t}-f_{x y} f_{y t}\right)^{2}=0
$$

under the transformation (4.16).

It is remarkable that the new finite dimensional models (4.8) and (4.9), the infinite dimensional systems (4.15) and (4.19) are expected to be integrable, particularly in the sense of possessing infinitely many symmetries and conservation laws. It is also shown in Ref. [23] that the finitedimensional models constructed in this way were completely integrable in the Liouville sense. However, the more detailed integrable properties need further investigations.

\section{Similarity solution from the nonlocal symmetry}

In order to make it easier to explore similarity solutions from the nonlocal symmetry (2.6), we first transform the sinh-Gordon equation (1.1) into the polynomial form

$$
4 u^{\prime} u_{x t}^{\prime}-4 u_{x}^{\prime} u_{t}^{\prime}-u^{\prime 4}+1=0,
$$

by the transformation $u=2 \ln u^{\prime}$. Correspondingly, the BT (1.2)-(1.3) is converted into

$$
\begin{aligned}
& u_{x}^{\prime} v^{\prime}+u^{\prime} v_{x}^{\prime}+\lambda\left(u^{2}-v^{2}\right)=0 \\
& 4 \lambda\left(u_{t}^{\prime} v^{\prime}-u^{\prime} v_{t}^{\prime}\right)+u^{\prime 2} v^{\prime 2}-1=0
\end{aligned}
$$

with $v^{\prime}$ determined by

$$
4 v^{\prime} v_{x t}^{\prime}-4 v_{x}^{\prime} v_{t}^{\prime}-v^{\prime 4}+1=0
$$

where the primes are removed for simplicity in the remaining part. Now the nonlocal symmetry (2.6) can be expressed as

$$
\sigma=e^{p}
$$

with

$$
p_{x}=-2 \lambda \frac{u}{v}-\frac{v_{x}}{v}, \quad p_{t}=-\frac{u v}{2 \lambda}+\frac{v_{t}}{v},
$$

and can be localized by introducing an auxiliary function $q$ determined by

$$
q_{x} v+2 \lambda e^{p}=0, \quad 2 \lambda q_{t}+v e^{p}=0 .
$$


In other words, the nonlocal symmetry (5.5) is localized for an extended sinh-Gordon system of Eqs. (5.1)-(5.7), which has the following general Lie point symmetry

$$
\left(\begin{array}{c}
\sigma^{u} \\
\sigma^{v} \\
\sigma^{p} \\
\sigma^{q} \\
\sigma^{\lambda}
\end{array}\right)=\left(\begin{array}{c}
\left(-c_{1} x+c_{3}\right) u_{x}+\left(c_{1} t+c_{2}\right) u_{t}-c_{4} e^{p} \\
\left(-c_{1} x+c_{3}\right) v_{x}+\left(c_{1} t+c_{2}\right) v_{t} \\
\left(-c_{1} x+c_{3}\right) p_{x}+\left(c_{1} t+c_{2}\right) p_{t}-c_{4} q-c_{5} \\
\left(-c_{1} x+c_{3}\right) q_{x}+\left(c_{1} t+c_{2}\right) q_{t}-\frac{1}{2} c_{4} q^{2}-c_{5} q-c_{6} \\
-c_{1} \lambda
\end{array}\right)
$$

obtained by requiring the extended sinh-Gordon system form invariant under the infinitesimal transformations $u \rightarrow u+\varepsilon \sigma^{u}, v \rightarrow v+\varepsilon \sigma^{v}, p \rightarrow p+\varepsilon \sigma^{p}, q \rightarrow q+\varepsilon \sigma^{q}, \lambda \rightarrow \lambda+\varepsilon \sigma^{\lambda}$ with an infinitesimal parameter $\varepsilon$.

Similarity solutions can be readily found by solving the symmetry constraint equations $\sigma^{i}=0$ $(i=u, v, p, q, \lambda)$ according to the standard procedure of the classical Lie group approach. It is seen that $c_{1}=0$ for nonzero $\lambda$ from $\sigma^{\lambda}=c_{1} \lambda=0, c_{2}$ and $c_{4}$ can be set as 1 without loss of generality.

The first type of similarity solution is given by

$$
\begin{aligned}
u & =\frac{1}{a} e^{P} \tanh (a(Q+t))+U, \\
v & =V \\
p & =-2 \ln (\cosh (a(Q+t))+P, \\
q & =-2 \tanh \left(a(Q+t)-c_{5}\right.
\end{aligned}
$$

where $a=\sqrt{c_{5}^{2}-2 c_{6}} \neq 0, U, V, P$ and $Q$ are group invariant functions of the group invariant variable $\xi=x-c_{3} t$, and are determined by the similarity reduction equations

$$
\begin{aligned}
U & =-\frac{4 c_{3} \lambda V_{\xi}}{4 \lambda^{2} c_{3}+V^{2}}, \\
P & =\ln \frac{4 a^{2} \lambda V}{4 \lambda^{2} c_{3}+V^{2}}, \\
Q & =\int \frac{4 \lambda^{2}}{4 \lambda^{2} c_{3}+V^{2}} \mathrm{~d} \xi, \\
V_{\xi}^{2} & =-\frac{1}{4 c_{3}} V^{4}-\frac{\left(16 c_{3}^{2} \lambda^{4}-16 a^{2} \lambda^{2}+1\right)}{16 c_{3}^{2} \lambda^{2}} V^{2} .
\end{aligned}
$$

In this case, the similarity solution of the sinh-Gordon equation (5.1) reads

$$
u=\frac{4 \lambda}{4 \lambda^{2} c_{3}+V^{2}}\left[a V \tanh \left(\int \frac{4 a \lambda^{2}}{4 \lambda^{2} c_{3}+V^{2}} \mathrm{~d} \xi+a t\right)+c_{3} V_{\xi}\right]
$$

with $V$ determined by Eq. (5.16).

In the case of $c_{6}=c_{5}^{2} / 2$, one can find the second similarity solution of the sinh-Gordon equation (5.1) in the form of

$$
u=-\frac{4 \lambda}{4 \lambda^{2} c_{3}+V^{2}}\left[c_{3} V_{\xi}+V\left(\int \frac{V^{2} \lambda^{2}}{\lambda^{2} V^{2}+1} \mathrm{~d} \xi+t\right)^{-1}\right]
$$

where $V$ is given by Eq. (5.16) with $a=0$, and $\xi=x-c_{3} t$. 
It is remarkable that the above group invariant solutions (5.17) and (5.18) can describe wave interactions between solitons and periodic waves in terms of Jacobi elliptic functions determined by the nonlinear equation (5.16) with and without $a$, respectively, similar to the interacting wave solutions obtained in Ref. [10,12,23,30] .

\section{Summary and discussions}

In summary, nonlocal symmetries are obtained by solving the linearized system constituted from the linearization of the SG equation with its BT. The most distinguished feature of the nonlocal symmetries is that they involve two solutions of the ShG equation stemming from the BT. Due to the intrusion of the parameter originated from the BT into the nonlocal symmetries, infinitely many nonlocal symmetries can be easily constructed by means of the parameter expansion procedure.

Obviously, some auxiliary functions are introduced when solving the nonlocal symmetries, and all these functions should satisfy some consistent conditions. It is easy to find that these conditions could be readily expressed in a divergence form, which is known as the conservation law. Therefore, nonlocal conservation laws are obtained in this way, and infinitely many nonlocal conservation laws are constructed similarly via the method of the parameter expansion.

Taking the obtained nonlocal symmetries as symmetry constraint conditions applied to any one equation of the BT, some new finite and infinite dimensional systems of $N$ coupled nonlinear equations can be produced. Though the integrability of these new systems is not proved in any sense, it is believed that they might possess many nice integrable properties, which need further investigations.

Finally, one of the nonlocal symmetry is localized for the sinh-Gordon equation in a polynomial form, and thus two kinds of similarity solutions are obtained. How to localize other nonlocal symmetries and then to obtain more group invariant solutions is still an open question.

\section{Acknowledgments}

The authors acknowledge the financial support by the National Natural Science Foundation of China (No. 11275123, 11475052, and No. 11675055).

\section{References}

[1] G. W. Bluman, A. F. Cheviakov and S. C. Anco, Applications of Symmetry Methods to Partial Differential Equations (New York, Springer, 2010).

[2] G. W. Bluman and S. Kumei, Symmetries and Differential Equations (Berlin, Springer, 1989).

[3] G. W. Bluman and A. F. Cheviakov, Framework for potential systems and nonlocal symmetries: Algorithmic approach, J. Math. Phys. 46 (2005) 123506.

[4] G. W. Bluman, A. F. Cheviakov and N. M. Ivanova, Framework for nonlocally related partial differential equation systems and nonlocal symmetries: Extension, simplification, and examples, J. Math. Phys. 47 (2006) 113505.

[5] A. F. Cheviakov and G. W. Bluman, Multidimensional partial differential equation systems: Generating new systems via conservation laws, potentials, gauges, subsystems, J. Math. Phys. 51 (2010) 103521.

[6] A. F. Cheviakov and G. W. Bluman, Multidimensional partial differential equation systems: Nonlocal symmetries, nonlocal conservation laws, exact solutions, J. Math. Phys. 51 (2010) 103522.

[7] P. A. Clarkson and M. D. Kruskal, New similarity reductions of the Boussinesq equation J. Math. Phys. 30 (1989) 2201-2213.

[8] F. Galas, New non-local symmetries with pseudopotentials, J. Phys. A: Math. Gen. 25 (1992) L981L986. 
[9] P. Górka and E. G. Reyes, The Modified Camassa Holm Equation, Int. Math. Res. Notices 2011 (2011) 2617-2649.

[10] X. N. Gao, S. Y. Lou and X. Y. Tang, Bosonization, singularity analysis, nonlocal symmetry reductions and exact solutions of supersymmetric KdV equation, J. High Energy Phys. 05 (2013) 029.

[11] R. H. Heredero and E. G. Reyes, Geometric Integrability of the Camassa-Holm Equation. II, International Mathematics Research Notices 2012 (2012) 3089-3125.

[12] X. R. Hu, S. Y. Lou and Y. Chen, Explicit solutions from eigenfunction symmetry of the Korteweg-de Vries equation, Phys. Rev. E 85 (2012) 056607.

[13] B. Konopelchenko and W. Strampp, The AKNS hierarchy as symmetry constraint of the KP hierarchy, Inverse Problems 7 (1991) L17-L24.

[14] I. S. Krasilshchik and A. M. Vinogradov, Nonlocal trends in the geometry of differential equations: symmetries, conservation laws, and Bäcklund transformations, Acta. Appl. Math. 15 (1989) 161-209.

[15] S. Y. Lou, A note on the new similarity reductions of the Boussinesq equation, Phys. Lett. A 151 (1990) 133-135.

[16] S. Y. Lou, Symmetries of the KdV equation and four hierarchies of the integrodifferential KdV equation, J. Math. Phys. 35 (1994) 2390-2396.

[17] S. Y. Lou and X. B. Hu, Nonlocal symmetries via Darboux transformations, J. Phys. A: Math. Gen. 30 (1997) L95-L100.

[18] S. Y. Lou and X. B. Hu, Infinitely many Lax pairs and symmetry constraints of the KP equation, $J$. Math. Phys. 38 (1997) 6401-6427.

[19] S. Y. Lou, (2+1)-dimensional integrable models from the constraints of the KP equation, Commun. Theor. Phys. 27 (1997) 249-252.

[20] S. Y. Lou, L. L. Chen and Q. x. Wu, New symmetry constraints of the modified KP equation, Chin. Phys. Lett. 14 (1997) 1-4.

[21] S. Y. Lou, X. Y. Tang and J. Lin, Similarity and conditional similarity reductions of a (2+1)-dimensional KdV equation via a direct method, J. Math. Phys. 41 (2000) 8286-8303.

[22] S. Y. Lou and H. C. Ma, Finite symmetry transformation groups and exact solutions of Lax integrable systems, Chaos, Solitons and Fractals 30 (2006) 804-821.

[23] S. Y. Lou, X. R. Hu and Y. Chen, Nonlocal symmetries related to Bäcklund transformation and their applications, J. Phys. A: Math. Theor. 45 (2012) 155209.

[24] W. X. Ma, New finite-dimensional integrable systems by symmetry constraint of the KdV equations, $J$. Phys. Soc. Jpn. 64 (1995) 1085-1091.

[25] E. Noether, Invariant variations problems, Trans. Theor. Stat. Phys. 1 (1971) 183-207.

[26] P. J. Olver, Applications of Lie Groups to Differential Equations (New York, Springer, 1993).

[27] W. Oevel and W. Schief, Squared eigenfunctions of the (modified) KP hierarchy and scattering problems of Loewner type, Rev. Math. Phys. 6 (1994) 1301-1338.

[28] A. G. Rasin and J. Schiff, The Gardner method for symmetries, J. Phys. A: Math. Theor. 46 (2013) 155202.

[29] E. G. Reyes, Nonlocal symmetries and the Kaup-Kupershmidt equation, J. Math. Phys. 46 (2005) 073507.

[30] X. Y. Tang, Z. F. Liang and J. Y. Wang, Nonlocal topological solitons of the sine-Gordon equation, J. Phys. A: Math. Theor. 48 (2015) 285204.

[31] A. M. Vinogradov and I. S. Krasilshchik, A method of calculating higher symmetries of nonlinear evolutionary equations, and nonlocal symmetries, Dokl. Akad. Nauk SSSR 253 (1980) 1289-1293.

[32] J. Y. Wang, X. Y. Tang, Z. F. Liang and S. Y. Liang, Infinitely many nonlocal symmetries and conservation laws for the (1+1)-dimensional Sine-Gordon equation, J. Math. Anal. Appl. 421 (2015) 685-696. 